\title{
Coronal plumes and the expansion of pressure-balanced structures in the fast solar wind
}

\author{
Sara Casalbuoni ${ }^{1,2}$, Luca Del Zanna ${ }^{3,4}$, Shadia R. Habbal ${ }^{5}$, and Marco Velli ${ }^{6,7}$
}

Abstract. The expansion of a coronal hole filled with a discrete number of higherdensity filaments (plumes) in overall pressure balance with the ambient medium is described within the thin flux tube approximation. The resulting solar wind model extends the results of Parker [1964] and Velli et al. [1994] to nonisothermal temperature profiles and includes a flux of Alfvén waves propagating both inside and outside the structures. Remote sensing and solar wind in situ observations are used to constrain the parameter range of our study. Close to the Sun, the precise plasma parameters are fundamental in determining the relative position of the critical points, which are found by means of an iterative procedure because the flows from the two regions are coupled. At greater distances the filling factor of the higher-density regions may vary largely, and streams which are either faster or slower than the wind arising from the ambient hole may result, depending on the temperature differences and on the flux of Alfvén waves assumed in the two regions. Velocity differences of the order of $\sim 50 \mathrm{~km} \mathrm{~s}^{-1}$, such as those found in microstreams in the high-speed solar wind, might be thus easily explained by reasonable fluctuation amplitudes at the Sun, although the natural candidates for plumes at large heliocentric distances are more likely to be the so-called pressure-balanced structures.

\section{Introduction}

Observations of the solar corona in white light show an abundance of structure in the polar regions in the form of rays or plumes [e.g., Saito, 1965; Koutchmy, 1977; Fisher and Guhathakurta, 1995], where the electron density is higher by factors of $2-5$ with respect to the background. Such structures have also been observed as short spikes in the lower corona at EUV and $\mathrm{X}$-ray wavelengths [e.g., Bohlin et al., 1975; Ahmad and Withbroe, 1977; Ahmad and Webb, 1978; Walker et al., 1993], and they have been recently the subject of coordi-

\footnotetext{
${ }^{1}$ Dipartimento di Astronomia e Scienza dello Spazio, University of Florence, Florence.

${ }^{2}$ Now at Physikalisches Institut, University of Bern, Bern.

${ }^{3}$ International Space Science Institute, Bern.

${ }^{4}$ Now at Dipartimento di Astronomia e Scienza dello Spazio, University of Florence, Florence.

${ }^{5}$ Harvard-Smithsonian Center for Astrophysics, Cambridge Massachusetts.

${ }^{6}$ Jet Propulsion Laboratory, California Institute of Technology, Pasadena.

${ }^{7}$ Permanently at Dipartimento di Astronomia e Scienza dello Spazio, University of Florence, Florence.
}

Copyright 1999 by the American Geophysical Union.

Paper number 1999JA900047.

0148-0227/99/1999JA900047\$09.00 nated observations by ground-based and Solar and Heliospheric Observatory (SOHO) spacecraft instruments [DeForest et al., 1997]: in this comprehensive study, plumes were observed all the way from the photosphere out to 15 solar radii, confirming the relationship between white-light and EUV density enhancements.

Plumes appear to originate above intense magnetic flux concentrations a few thousand kilometers in diameter (although not all flux concentrations have overlying plumes) and to expand initially very rapidly with height in a way consistent with potential or force-free magnetic field models [Del Zanna et al., 1997, and references therein; see also Del Zanna et al., 1998a; Suess et al., 1998]. Above $\sim 2 \times 10^{4} \mathrm{~km}$ the rapid expansion terminates and plumes should undergo the general coronal hole expansion, although it is not clear whether plumes expand even faster than that (see Fisher and Guhathakurta [1995] and DeForest et al. [1997] for contrasting observations). This expansion concerns the actual cross-sectional area of the flux tube itself, and it must not be confused with the fact that plumes are also seen to diverge superradially, in the sense that their direction meets the polar axis in a point located between the Sun's center and the coronal hole surface. This is another clear indication that plumes follow the general magnetic field pattern. At a given flux concentration on the Sun, the presence or absence of an overlying plume seems to depend on the complexity of the local magnetic 
field topology, plumes appearing preferentially where minority-polarity fields impinge on the dominant flux concentrations [Wang et al., 1997]. This observation appears to substantiate phenomenological models for plume formation in which the dynamics and enhanced heating induced by magnetic reconnection give rise to the increased density in the plumes [Velli et al., 1994; Wang, 1994; Wang and Sheeley, 1995].

The precise temperature structure of plumes is much less well understood, though indirect evidence via density gradients determined from polarization brightness measurements (i.e., scale height temperatures) [Fisher and Guhathakurta, 1995] or via OVI line broadenings [Hassler et al., 1997] seems to indicate that the plasma in plumes, at a given height in the lower corona, is cooler by as much as $30 \%$ with respect to the background medium. In any case, line ratios show a lower electron temperature in plumes [DeForest et al., 1997; Wilhelm et al., 1998]. What is not clear is whether plumes are really cooler at all heights, as will be discussed in section 3.

It is unknown how the structures observed in the corona merge to yield the remarkably homogeneous high-speed polar solar wind observed by the Ulysses spacecraft, though a number of models for the extension of plume-like structures into the solar wind have been presented [Parker, 1964; Velli et al., 1994; Wang, 1994; Habbal et al., 1995]. In particular, it is of interest to determine whether the residual oscillations in radial velocity observed at Ulysses, the so-called microstreams [Neugebauer et al., 1995], or alternatively the rarer pressure-balanced structures [Thieme et al., 1990; McComas et al., 1995, 1996; Poletto et al., 1996], are related to the coronal hole structures observed near the Sun.

Parker [1964] was the first to describe the extension of a filamentary corona into the interplanetary medium, and he essentially concluded that coronal structures would rapidly inflate and fill the whole heliosphere. Velli et al. [1994] extended Parker's model and considered denser plumes in a nonempty background coronal hole, solving the coupled flow equations with an assumed pressure balance. An isothermal corona was considered, with a temperature difference between the plume and background hole plasma. In such a model, the temperature difference has an immediate consequence on the difference in asymptotic flow speed, hotter plasma flowing necessarily faster. Plume-like structures were shown to remain collimated out to tens of solar radii, before fanning out once the ratio of plasma to magnetic pressures attained values close to unity.

Subsequent models of the solar wind flow inside and outside plumes [Wang, 1994; Habbal et al., 1995] have neglected the coupling of the plume and coronal hole plasma required by an approximate pressure balance. On the other hand, the wind modeling was much more sophisticated in the sense that the energy equation was considered and parameter ranges were explored so as to satisfy observational requirements both at the coronal base and at $1 \mathrm{AU}$. In all cases, energy deposition was parameterized by exponential heating functions localized at different heights, and a flux of Alfvén waves was included in the momentum equation; the results showed that for comparable values of the heating rates, the denser plumes have a lower peak temperature and lower velocities. In particular, Wang showed that an extra heating just above the coronal base, possibly released via magnetic reconnection from the interacting fields below, is an essential feature for the formation of the plume. Most of this heating is radiated away locally, but its presence is able to account for the enhanced density in plumes.

In this paper we further develop the model of expanding filamentary pressure-balanced structures of Parker [1964] and Velli et al. [1994]. We will consider supraspherically expanding coronal hole geometries and nonisothermal temperature profiles, and we will include a flux of Alfvén waves both in coronal plumes and in the ambient hole. Since our model is strongly focused on the transverse dynamics and given the large uncertainty in the energetics of the coronal expansion, we prefer to omit the energy equation entirely and to consider a given parameterized set of temperature profiles which nicely fits both observational and contemporary simulated temperatures in the corona and solar wind. In section 2 we return to the earlier models of Parker and Velli et al. to explain the basic mechanisms of plume expansion in the isothermal approximation, including the effects of supraspherical coronal hole expansion. We will show that Parker's original conclusion was incorrect and that a filamentary corona with a large-scale open magnetic field would, in the absence of instabilities or mixing and compressive effects due to solar rotation, maintain its structure out to large radial distances. In section 3 we will introduce a family of temperature profiles, which gives a good fit of recent inferred coronal and in situ measured temperature profiles, and we will use these to calculate the expansion of plumes and ambient holes in pressure balance. We will also consider an undamped Alfvén wave flux both in the hole and in the plume plasma and discuss a plausible model for the amplitude and structure of the expanding plumes. In section 4 we will relate our models to the observed properties of the inner corona and fast solar wind streams, discussing in particular the possible relationship of plumes with the inhomogeneities found by Helios and Ulysses, namely, pressure-balanced structures and microstreams.

\section{Isothermal Plumes}

In this section the problem of the expansion of dense filamentary structures in the solar wind will be tackled by using the same mathematical formalism and phys- 
ical approximations as those in the previous works by Parker [1964] and Velli et al. [1994]. We will consider initially only the isothermal case, with the aim of a better understanding of the physics of the coupled streaming regions. Although the isothermal approximation is certainly unrealistic for the coronal and interplanetary plasma, this simple case will enable us to draw some important physical conclusions, which will remain valid also when a more realistic temperature profile is employed (see section 3 ).

\subsection{Governing Equations and Method of Solution}

Consider a coronal hole made up of two different components: a background low-density region, whose dynamical and thermodynamical variables will be labeled by the index 1 , and a discrete number of denser structures, whose average observables will be labeled by the index 2. Both these interplume and plume regions will be treated as one-dimensional (1-D) thin (or slender) flux tubes, with all dynamical variables depending on heliocentric distance $r$ alone. In this approximation, curvature terms and effects due to solar rotation are neglected, and all variables are assumed to have constant values on flux tube cross sections, whose area varies with $r: A_{i}=A_{\imath}(r)$. These assumptions are better satisfied near the polar axis, where the field lines are mainly radial, while at the coronal hole's boundaries, plumes are tilted toward the equator. Nevertheless, here the particular geometrical distribution of dense flux tubes inside the coronal hole is not important, since the only information concerning the geometry of plumes which enters the model is the base filling factor $\alpha_{2}$, i.e., the percentage of the coronal hole surface occupied by plumes.

Following Parker [1964] and Velli et al. [1994], we will assume here that mass and magnetic fluxes are conserved separately in the two regions, so that the MHD equations, for $i=1,2$, read

$$
\begin{aligned}
B_{\imath} A_{i} & =B_{i 0} A_{i 0} \\
n_{i} u_{i} A_{i} & =n_{\imath 0} u_{\imath 0} A_{i 0} \\
m n_{i} u_{i} \frac{d u_{i}}{d r}= & -\frac{d p_{\imath}}{d r}-\frac{G M_{\odot}}{r^{2}} m n_{i} \\
p_{i} & =\frac{k}{\mu} n_{i} T_{i} .
\end{aligned}
$$

Except where specified, the plasma is considered to be made up of totally ionized hydrogen, thus the mean molecular mass is $\mu=0.5$ ( $m$ is the proton mass and $n_{\imath}$ is the electron number density). The temperature $T_{i}$, constant at this stage, must be interpreted as the average value of the electron and proton temperatures, whereas $p_{\imath}$ is the total gas pressure. The index 0 indicates values at some initial radius $r_{\min }$, which may be called the coronal base, where the magnetic field is sup- posed to have assumed already a simple 1-D structure (we will assume $r_{\min } \equiv R_{\odot}$ for simplicity).

The continuity and momentum equations along the magnetic field for the plume and interplume plasmas are coupled by constraints which must hold across flow tubes: the first is the conservation of the total area in the expanding coronal hole, which may be written as $\left(s_{0}=1\right)$ :

$$
A_{1}+A_{2}=A_{\text {tot }} \equiv A_{\text {tot } 0} s,
$$

where the overall coronal hole expansion rate $s$ is a given function of $r\left(s \sim r^{2}\right.$ in the simple spherical geometry). The second constraint describes the total lateral pressure (kinetic and magnetic) balance, which is the momentum equation integrated across the plumeinterplume boundaries in the thin flux tube approximation:

$$
p_{1}+\frac{B_{1}^{2}}{8 \pi}=p_{2}+\frac{B_{2}^{2}}{8 \pi} .
$$

Let us now nondimensionalize the physical quantities with respect to some typical values at the coronal base, for example, $B_{\star}=1 \mathrm{G}, n_{\star}=10^{8} \mathrm{~cm}^{-3}$, $T_{\star}=10^{6} \mathrm{~K}$, and $u_{\star}=\sqrt{k T_{\star} / \mu m}=128 \mathrm{~km} \mathrm{~s}^{-1}$. If we also choose $r / R_{\odot} \rightarrow r, A_{i} / A_{i 0}=s a_{i}, \alpha_{i}=$ $A_{i 0} / A_{\text {tot } 0}, \beta_{\star}=\left(k n_{\star} T_{\star} / \mu\right) /\left(B_{\star}^{2} / 8 \pi\right)=0.69$, and $g_{\star}=$ $G M_{\odot} \mu m / R_{\odot} k T_{\star}=11.6$, the equations take the following form $(i=1,2)$ :

$$
\begin{gathered}
B_{\imath}=\frac{B_{i 0}}{s a_{i}}, \quad n_{i} u_{i}=\frac{n_{i 0} u_{i 0}}{s a_{i}}, \\
\left(\frac{u_{\imath}^{2}}{T_{i}}-1\right) \frac{u_{i}^{\prime}}{u_{\imath}}=\frac{s^{\prime}}{s}+\frac{a_{\imath}^{\prime}}{a_{\imath}}-\frac{g_{\star}}{T_{i} r^{2}} \\
\alpha_{1} a_{1}+\alpha_{2} a_{2}=1 \\
B_{1}^{2}+\beta_{\star} n_{1} T_{1}=B_{2}^{2}+\beta_{\star} n_{2} T_{2},
\end{gathered}
$$

where primes indicate radial derivatives.

This set of equations may be rewritten as a system of four coupled equations in the four unknowns $u_{1}, u_{2}$, $a_{1}$, and $a_{2}$. The straightforward solution would be to diagonalize the system (by also differentiating (9) and (10)) and to integrate it by using a standard numerical method for systems of ordinary differential equations (ODEs) with given initial conditions. However, since the wind solutions in both regions are required to be transonic, the base values of the two velocities must be determined by the solution itself in order to remove the singularities present in the system. Thus standard solution techniques can only be applied beyond the critical points.

Owing to the coupling between the two regions, the positions of the critical points, which are determined by setting the right-hand side of (8) to zero (for $s=r^{2}$ and in the uncoupled case $a_{i}=1$, the critical points positions are given by $r_{c}=g_{\star} / 2 T_{i}$ ), are not known a priori, and following Velli et al. [1994], an iterative technique is employed: equation (8) is first written in terms of 

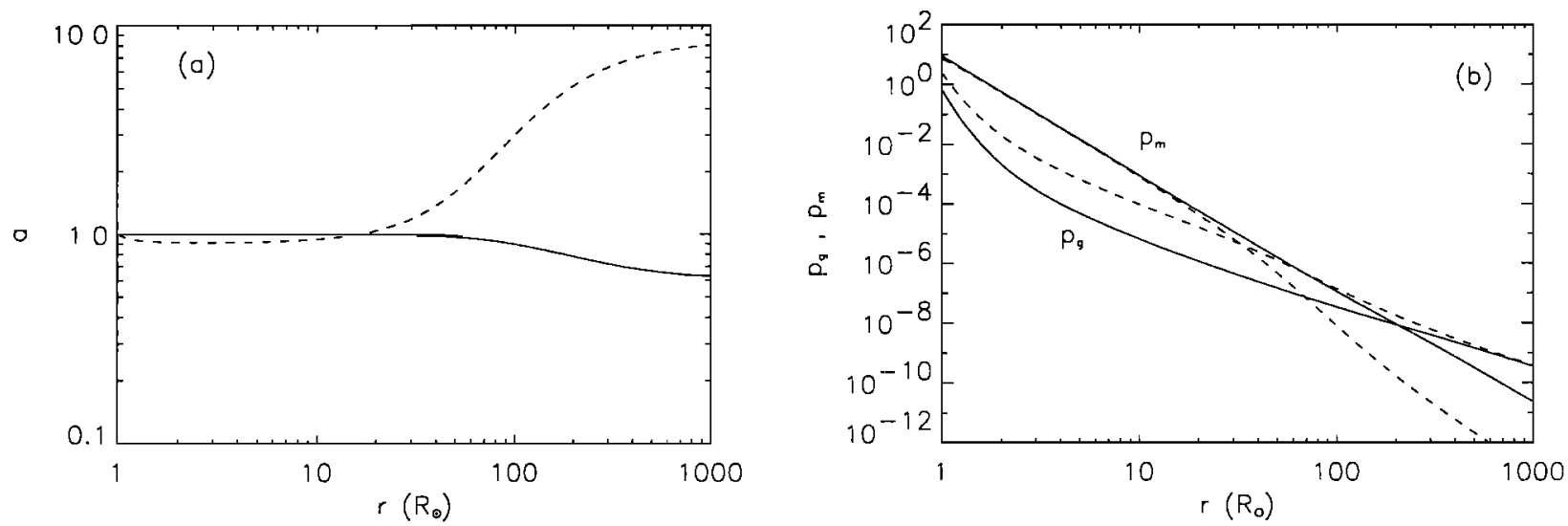

Figure 1. (a) Nonradial expansion $a(r)$ (which is the normalized filling factor) for the interplume (solid line) and the plume (dashed line) regions. Here the initial filling factor of the plumes is $\alpha_{2}=5 \%$. The other values are $n_{10}=10^{8} \mathrm{~cm}^{-3}, n_{20}=3 \times 10^{8} \mathrm{~cm}^{-3}, T_{1}=10^{6} \mathrm{~K}$, and $T_{2}=1.2 \times 10^{6} \mathrm{~K}$ (hotter and denser plume). Note the initial contraction of plumes before the definitive expansion. (b) The normalized magnetic and gas pressures for the same set of parameters (magnetic pressure curves are those starting with larger values at the base).

$u_{i}$ and $u_{i}^{\prime}$ alone; then a standard shooting method is applied to solve for one velocity, using values for the other calculated at the previous iteration step. In the first iteration we solve for the interplume region as if there were no plumes (corresponding to the uncoupled case with $\alpha_{2}=0$ ); then the method is iterated until convergence is achieved. Clearly, convergence is faster for smaller values of $\alpha_{2}$.

\subsection{Results}

Consider, for example, a plume which is 3 times denser than the background coronal hole (at the base) (e.g., $n_{10}=1$ and $n_{20}=3$ in the usual units of $n_{\star}=10^{8} \mathrm{~cm}^{-3}$ ), and also $20 \%$ hotter (e.g., $T_{1}=1$ and $\left.T_{2}=1.2\left(\times 10^{6} \mathrm{~K}\right)\right)$. The base interplume magnetic field is taken to be $B_{10}=3$ (in Gauss), while $B_{20}$ is to be determined from (10). The initial filling factor of plumes is $\alpha_{2}=5 \%\left(\alpha_{1}=1-\alpha_{2}=95 \%\right)$, and spherical overall expansion is assumed $\left(s=r^{2}\right)$. The resulting normalized filling factors and the gas and magnetic pressures are shown in Figure 1.

The plume expands approximately radially within $\sim 20 R_{\odot}$; then $a_{2}$ starts to increase, and at the Earth's orbit the filling factor of plumes reaches a value as large as $\alpha_{2} a_{2} \sim 27 \%$. Also, note that the plasma $\beta$ reaches unity at $r \sim 30 R_{\odot}$ in plumes and at $r \sim 200 R_{\odot}$ outside. The effects of the coupling between the two regions clearly increases together with the plasma $\beta$, confirming what was found by Velli et al. [1994] and in the two-dimensional low- $\beta$ model by Del Zanna et al. [1997]. The initial slight contraction and further expansion of plumes (relative to the overall $r^{2}$ expansion of the coronal hole) are easily explained: near the base, where pressure balance is imposed, densities drop faster than $B^{2}$ (the decay is exponential), and the magnetic pressure thus dominates, so that there is a compression by the stronger external field. When the $\beta$ increases, the situation is reversed: the higher gas pressure inside the plume pushes the magnetic field lines outward and the plume fans out until this expansion (and the corresponding compression of the interplume region) smoothes the gas pressure differences in the two regions, as can be seen in Figure $1 b$.

The superradial expansion of plumes $a_{2}$ is the greatest for negligible base filling factors, while it is reduced for larger values of $\alpha_{2}$; the filling factor as a whole, $\alpha_{2} a_{2}$, however, remains a monotonically increasing function of $\alpha_{2}$. For example, if plumes occupy the $20 \%$ of the coronal hole surface, the corresponding value at $1 \mathrm{AU}$ is $\alpha_{2} a_{2}=59 \%$.

Another interesting aspect is that plumes tend to have a faster plasma flow, as long as the temperature is greater than or equal to that of the adjacent interplume plasma. In the run with $T_{2}=1.2$ the velocities at the Earth's orbit are $u_{1}=473$ and $u_{2}=607 \mathrm{~km} \mathrm{~s}^{-1}$, respectively. If we keep all other conditions the same but take an isothermal plasma everywhere, $T_{1}=T_{2}=1$, the final velocities are $u_{1}=481$ and $u_{2}=503 \mathrm{~km} \mathrm{~s}^{-1}$. Note that this shear is entirely due to the coupling between the two streaming regions, since noninteracting Parker-type flows with the same temperatures would have the same velocity profiles, regardless of the different densities. The reason for the excess acceleration is the decreasing plume-interplume density contrast due to pressure balance that at large distances where the magnetic contribution is negligible, requires $n_{1} / n_{2} \rightarrow 1$. This can be shown in a quantitative way by integrating (8), or better (3) directly, separately for the plume and interplume media. Since at the solar surface the pressure balance constraint does not play a great role (because of the dominance of magnetic pressure), one 
may safely neglect differences in the initial flow speeds (if the temperature is the same, $T_{1}=T_{2}=T$ ) and then subtract the two integrated equations to obtain

$$
u_{2}^{2}-u_{1}^{2} \simeq 2 T \log \left(\frac{n_{20}}{n_{10}} \frac{n_{1}}{n_{2}}\right)
$$

which proves that velocity shears are small as long as the density ratio remains nearly constant, whereas the plume will be faster than the interplume farther out.

When the temperatures are the same, the increase in the filling factor is also very much reduced, with a final value of only $\alpha_{2} a_{2}=8 \%$, compared with the previous $27 \%$ with the same $5 \%$ initial filling factor. The situation changes drastically if we consider cooler plumes. For $T_{2}=0.8$, which is $20 \%$ cooler than $T_{1}, a_{2}$ is always less than 1 , and the final filling factor at $1 \mathrm{AU}$ is just $3 \%$. The plume is always slower than the surrounding region, with values at $1 \mathrm{AU}$ of $u_{1}=483$ and $u_{2}=407 \mathrm{~km} \mathrm{~s}^{-1}$, and beyond $1.6 R_{\odot}$, the smaller scale height in plumes reduces the density to values below that in the interplume region, and thus cool plumes are both slower and less dense than the background coronal hole.

Consider now the effects of a superradial expansion of the coronal hole. White-light observatlons show clearly that the angle subtended by the coronal hole increases from $r=1 R_{\odot}$ to, say, $r=5 R_{\odot}$, following the profile of the boundaries of equatorial streamers, although this has been recently questioned by Ulysses radio occultation measurements [Woo and Habbal, 1997].

For the coronal hole expansion we use here the analytical function first proposed by Kopp and Holzer [1976] and the fit made by Munro and Jackson [1977], who gave the values of the parameters by using white-light data. The function is

$$
f(r)=\frac{f_{\max } \exp \left[\left(r-r_{1}\right) / \sigma\right]+f_{1}}{\exp \left[\left(r-r_{1}\right) / \sigma\right]+1}
$$

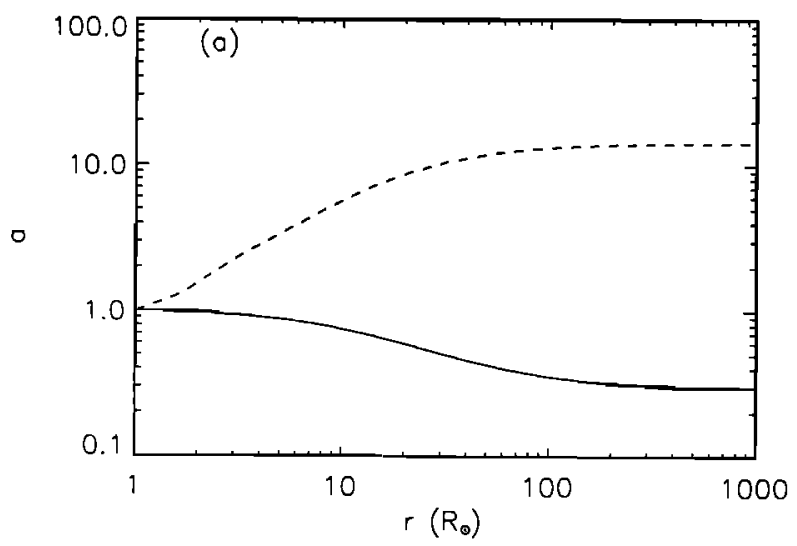

where $f_{1}=1-\left(f_{\max }-1\right) \exp \left[\left(1-r_{1}\right) / \sigma\right]$. The normalized area is given by $s(r)=r^{2} f(r)$, and the fit parameters are $f_{\max }=7.26, r_{1}=1.31$, and $\sigma=0.51$. Note that the nonradial expansion occurs in the range $\left|r-r_{1}\right|<\sigma$. From the expression for the coronal hole area $A_{\text {tot }}$ we can derive the relation between the function $f(r)$ and the angle $\theta(r)$ subtended by the coronal hole boundary with the adjacent streamer (for an axisymmetric configuration):

$$
A_{\text {tot }}=2 \pi r^{2}[1-\cos \theta(r)] \Rightarrow f=\frac{1-\cos \theta(r)}{1-\cos \theta_{0}},
$$

where $\theta_{0}$ is the colatitude of the coronal hole boundary at the base. For an initial area $A_{\text {toto }}=0.52$, which corresponds to $\theta_{0}=23.5^{\circ}$, the given value of $f_{\max }$ yields a final colatitude of $\theta=66.4^{\circ}$. Note that a coronal hole with the same initial area fills the entire hemisphere if $f_{\max } \approx 12$.

In Figure 2 we show the same quantities as those in Figure 1 for the same set of parameters, but this time we include the superradial coronal hole expansion. The expansion of the plume region now occurs right from the start, with no initial contraction, and to a much greater extent, with plumes occupying $70 \%$ of the total coronal hole flux tube section at $1 \mathrm{AU}$ (with an increase in the filling factor of $a_{2}=14$ times the initial value). This is due to the rapid drop in the magnetic field strengths, which causes the gas pressure effects to take over much closer to the Sun with the consequent expansion of the high-density plume region.

This fast expansion has consequences on the other plasma parameters as well. For example, the velocity of plumes is much higher in the inner corona, with a base value of $u_{20}=18.5 \mathrm{~km} \mathrm{~s}^{-1}$ and a critical point at $2.2 R_{\odot}$, to be compared with the corresponding values of $u_{10} \sim 1$ and $6.3 R_{\odot}$ in the interplume region and with $u_{20} \sim 1$ and $4.7 R_{\odot}$ in the case of no superradial

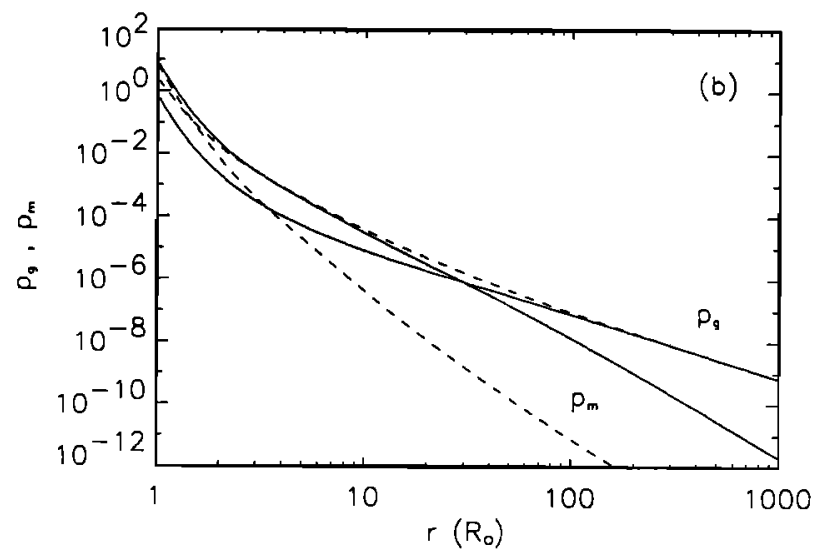

Figure 2. The same quantities as those shown in Figure 1 when the superradial coronal hole expansion is considered, including (a) the function $a_{i}(r)$ and (b) the magnetic and gas pressures. The values of all the other parameters are left unchanged. Note the faster drop in the magnetic pressures (Figure $2 \mathrm{~b}$, the two lines decaying faster at large distances) which causes a large and sudden expansion of the plume. The function that gives the expansion and the values used for its parameters are reported in the text. 
expansion $\left(f_{\max }=1\right)$. On the other hand, the final velocities are similar to the first run, as we find $u_{1}=446$ and $u_{2}=602 \mathrm{~km} \mathrm{~s}^{-1}$.

To conclude, we should point out that the effects of the coupling between the two regions due to the total pressure preservation across the boundary of the flux tubes, equation (10), are enhanced by the presence of a faster than radial coronal hole expansion provided the parameters at the base are the same. Most important of all are the initial values of the magnetic fields, which should be increased to yield the same values at $1 \mathrm{AU}$ when $f_{\max }>1$. This problem will be tackled in section 3 , where we try to build a more realistic wind model by considering the constraints provided by in situ measurements of the various plasma parameters.

\subsection{Comparison With Parker's Model for Dense Streamers}

The problem of the expansion of dense coronal structures into interplanetary space was first treated by Parker [1964], who considered the extreme case in which the gas density between streamers (to use Parker's terminology) was identically zero. In his model the thin flux tube approximation was also used; thus it is interesting to compare our numerical model with his analytical approach.

Parker [1964] assumed a negligible filling factor $\left(\alpha_{2}=\right.$ 0 ) for the dense structures and a spherical expansion for the overall coronal hole $(f=1)$, so that the background magnetic field always expands as $B_{1} \sim r^{-2}\left(a_{1}=1\right)$, and the pressure balance relation becomes

$$
\frac{B_{10}^{2}}{r^{4}}=\frac{B_{20}^{2}}{a_{2}^{2} r^{4}}+\beta_{\star} n_{2} T_{2} .
$$

Equation (14) for $a_{2}$ is coupled to the wind equation for $u_{2}$ through the density $n_{2}$ and the continuity equation. In order to solve the equations analytically, Parker decoupled them by choosing an approximate behavior for the density $n_{2}$. The hydrostatic profile

$$
n_{2}=n_{20} \exp \left[-\left(g_{\star} / T_{2}\right)(1-1 / r)\right]
$$

was employed by Parker everywhere; thus limiting the validity of his results to the subsonic region. This becomes apparent in Figure 3, where the relative angular width of the dense structure, proportional to $\sqrt{a_{2}}$ following Parker's notation, is plotted as a function of $\omega=r /\left(g_{\star} / T_{2}\right)$ for both his analytical model and our numerical work. The angular width is defined in Parker's paper as $\sqrt{Z(\omega) / \omega^{2}}$, where

$$
Z(\omega)=a_{2} r^{2} \sqrt{\frac{\exp \left(-g_{\star} / T_{2}\right)}{\nu^{2}-1}}, \quad \nu^{2}=\frac{B_{10}^{2}}{\beta_{\star} n_{20} T_{2}} .
$$

It is straightforward to verify that the use of (15) led Parker to the simple following function:

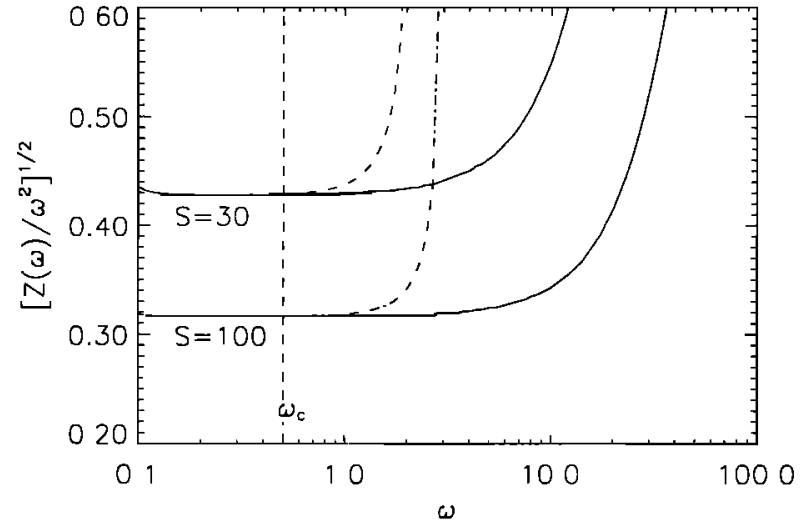

Figure 3. The angular width as a function of the dimensionless heliocentric distance $\omega$ (the critical point is at $\omega_{c}=0.5$, for $g_{\star} / T_{2}=10$ ). The parameter $S$, defined in the text, may be rewritten in terms of the plasma $\beta$ at the base of the dense structure, according to the relation $\beta_{20}=\left[S\left(g_{\star} / T_{2}\right)^{4} \exp \left(-g_{\star} / T_{2}\right)\right]^{-1}$ (a smaller $S$ means a stronger gas pressure and/or a weaker magnetic pressure in the streamer region and vice versa). Solid lines are the results of our numerical model, while dash-dotted lines represent Parker's analytical function, equation (17). Our results are obtained by taking typical coronal plasma values (temperature of $10^{6} \mathrm{~K}$ and number density of $10^{8} \mathrm{~cm}^{-3}$, so that $T_{2}=n_{20}=1$ ); $S=30$ corresponds to a base plasma $\beta$ of $7.92 \times 10^{-2}$, whereas $S=100$ corresponds to $2.25 \times 10^{-2}$.

$$
Z(\omega)=\frac{1}{\sqrt{S / \omega^{4}-\exp (1 / \omega)}}
$$

where the new free parameter $S$ is defined as $S=$ $\nu^{2}\left(g_{\star} / T_{2}\right)^{-4} \exp \left(g_{\star} / T_{2}\right)$, which clearly has a singularity, interpreted by Parker $[1964$, p. 690 , in the abstract] as an expansion of the streamers to fill the whole coronal hole, "no matter how small a fraction they may occupy at the Sun." However, from the comparison with our numerical results we can clearly see that the expansion predicted by Parker, which is boundless because the external plasma was neglected, occurs too close to the Sun (note the logarithmic scale).

The disagreement between the two models in the supersonic region is obviously due to the failure of the hydrostatic approximation beyond the critical point. In fact, the presence of a plasma outflow leads to an additional decay in the density $n_{2}$, which in the case of uncoupled flux tubes would amount to a factor $\exp \left[-\left(u_{2}^{2}-\right.\right.$ $\left.u_{20}^{2}\right) / 2 T_{2}$ ], causing a tangible drop in the gas pressure and a consequent reduction of the streamer expansion.

\section{A Realistic Fast Wind Model: Nonisothermal Case}

In order to build a realistic fast solar wind model, the isothermal assumption must be relaxed. While the isothermal approximation allows one to understand 
more easily the physics of the interaction between the two streaming regions, any comparison with both remote sensing and in situ measurements of the plasma parameters can only be made if the temperature is not kept constant. As a matter of fact, both the electron and proton temperatures are seen to drop by 1 or even 2 orders of magnitude from the inner corona out to $1 \mathrm{AU}$.

A nonisothermal temperature profile, with a temperature maximum of $\sim 5 \times 10^{6} \mathrm{~K}$ in the corona, is also able to provide the acceleration required to reach the observed high speeds of $750-800 \mathrm{~km} \mathrm{~s}^{-1}$, which is always the main difficulty one faces when trying to build a fast solar wind model. A possible alternative is momentum deposition by linear Alfvén waves propagating along the flux tubes, which appears in the form of an additional pressure both in the momentum equation and in the total pressure balance. Contribution by Alfvén waves with wavelengths much shorter than typical scale heights will be included here, even though for presently estimated values of the amplitude they will produce only minor global effects. Such amplitudes, of order $10 \mathrm{~km} \mathrm{~s}^{-1}$ at the coronal base, are sufficient, however, to produce observable differences between plume and interplume flows.

\subsection{Modified Equations}

Because we do not solve an energy equation for the plasmas inside and outside plumes, the temperature profiles in the two regions $T_{i}(r)$ must be given a priori, taking into consideration as many observational and theoretical constraints as possible. From a mathematical point of view the only changes appear in the momentum equations, equation (8), where an extra term $-T_{i}^{\prime} / T_{i}$ must be added on the right-hand side.

The presence of linear Alfvén waves propagating along the flux tubes also introduces additional terms. The time-averaged fluctuations $\delta u \equiv<\delta u_{w}^{2}>^{1 / 2}$ and $\delta B \equiv<$ $\delta B_{w}^{2}>^{1 / 2}$ yield an additional isotropic wave pressure,

$$
p_{w}=\frac{(\delta B)^{2}}{8 \pi}=\frac{1}{2} \rho(\delta u)^{2},
$$

where the relation $\delta B / B=\delta u / v_{A}$ has been used $\left(v_{A} \equiv\right.$ $B / \sqrt{4 \pi \rho}$ is the Alfvén velocity). Note that these relations hold in the WKB limit, that is, when waves see the medium in which they propagate as homogeneous, hence only for wavelengths which are small compared with the typical length scale of the Alfvén speed variation, which coincides basically with the local gravitational scale height in the inner corona. This is a good approximation for the typical wave frequencies that are observed in the solar wind in situ in the ecliptic plane [Hollweg, 1973], although it may break down for the longest-period components ( $\geq 10$ hours) observed in the fast wind at high latitudes [Smith et al., 1995].

By using the conservation of the wave action density (the wave energy flux is not conserved in a moving medium), it may be shown [see Alazraki and Couturier,
1971; Jacques, 1977; Velli, 1993, and references therein] that the wave pressure evolves as

$$
p_{w}=p_{w_{0}} \frac{M_{A 0}\left(1+M_{A 0}\right)^{2}}{M_{A}\left(1+M_{A}\right)^{2}}
$$

where $M_{A} \equiv u / v_{A}=\sqrt{\beta_{\star} / 2} \sqrt{n} u / B \sim n^{-1 / 2}$ is the Alfvénic Mach number. After some algebra the nondimensional momentum and pressure balance equations (8) and (10) may be shown to become

$$
\begin{gathered}
{\left[\frac{u_{i}^{2}}{T_{i}}-\left(1+\tau_{i} \Omega_{i}\right)\right] \frac{u_{i}^{\prime}}{u_{i}}} \\
=\left(1+\tau_{i} \Omega_{i}\right)\left(\frac{s^{\prime}}{s}+\frac{a_{i}^{\prime}}{a_{2}}\right)-\frac{T_{i}^{\prime}}{T_{i}}-\frac{g_{\star}}{T_{i} r^{2}}, \\
B_{1}^{2}+\beta_{\star} n_{1} T_{1}\left(1+\Omega_{1}\right)=B_{2}^{2}+\beta_{\star} n_{2} T_{2}\left(1+\Omega_{2}\right),
\end{gathered}
$$

where $\Omega_{\imath}$ and $\tau_{\imath}$ are given by (the index $i$ is understood)

$$
\Omega \equiv \frac{p_{w}}{p} \sim \frac{1}{T} \frac{M_{A}}{\left(1+M_{A}\right)^{2}}, \quad \tau=\frac{1+3 M_{A}}{2\left(1+M_{A}\right)},
$$

and the relation $\delta u=\sqrt{2 \Omega T}$ holds in nondimensional units. The net effect of the presence of waves $(\Omega>0)$ is to accelerate the wind, since the position of the critical point moves closer to the Sun's surface, and at the same time the value of the velocity at the critical point is increased.

\subsection{Temperature Profile}

As stated in section 1, observations of coronal hole and solar wind temperatures are needed to constrain the otherwise arbitrary analytical profiles. Unfortunately, remote measurements of temperatures in coronal holes are extremely difficult to make and are affected by many sources of error, since all the techniques can infer temperatures only indirectly [Habbal et al., 1993].

However, coronal hole electron temperatures appear to be always in the range $T_{e} \sim 7 \times 10^{5}-1.6 \times 10^{6} \mathrm{~K}$ below $1.6 R_{\odot}$. Isotopic composition data from the solar wind ion composition spectrometer (SWICS) on Ulysses seem to indicate the presence of a maximum around $1.5 \times 10^{6} \mathrm{~K}$ below $2 R_{\odot}$ in the electron temperature profile [Geiss et al., 1995; Ko et al., 1997], although recent data by the solar ultraviolet measurements of emitted radiation (SUMER) instrument on SOHO show that $T_{e}<8 \times 10^{5} \mathrm{~K}$ below $1.6 R_{\odot}$, with the temperature in plumes decreasing to $3 \times 10^{5} \mathrm{~K}$ [Wilhelm et al., 1998]. Similar results, but mixing plume and interplume contributions, are found by David et al., [1997].

Until a few years ago it was generally accepted that in the inner corona, protons had temperatures of the same order of magnitude as electrons, $T_{p} \approx T_{e} \approx 10^{6} \mathrm{~K}$, but recent observations of Doppler broadenings of UV lines seem to indicate much higher kinetic temperatures for the protons and heavier ions $\left(\sim 4-6 \times 10^{6} \mathrm{~K}\right)$ [e.g., Kohl 
et al., 1996; Wilhelm et al., 1998]. This line broadening could also be partially due to nonthermal effects, such as a turbulent wave field, so such high values should be considered an upper limit for proton temperatures; in any case, the net result is always an additional pressure in the momentum equation. Here we will cumulate all these possible effects in our temperature $T$, which hence has the meaning of an effective temperature (the contribution by Alfvén waves, usually considered in the definition of the effective temperature, is treated here separately).

Another fundamental new result is that the acceleration to the final high-speed flow velocities happens within the first 10-20 solar radii [Grall et al., 1996] and that speeds as high as $150-200 \mathrm{~km} \mathrm{~s}^{-1}$ may be reached even at $2-3 R_{\odot}[$ Strachan et al., 1993; Corti et al., 1997; Kohl et al., 1997]. Theoretical fast solar wind models which consider high proton temperatures have already been produced [McKenzie et al., 1995; McKenzie et al., 1997; Esser et al., 1997; Hu et al., 1997] and seem to successfully yield a realistic high-speed wind already in the inner corona, whereas models where the wind is mainly driven by Alfvén waves at large distances fail to give the terminal speeds already at $\sim 10 \mathrm{AU}$ [Wang, 1994; Habbal et al., 1995]. For all these reasons, here we will use effective temperature profiles which have a large maximum (a few million degrees) near the Sun and then decrease to interplanetary values, in agreement with the models cited above. The final choice of the parameters defining the actual shape of the analytical function will come from comparisons of the densities predicted by the model with white-light observations within 6 solar radii and from all quantities measured at $1 \mathrm{AU}$.

Recent measurements of temperatures and temperature gradients in the inner heliosphere are provided essentially by two missions: Helios and Ulysses. In the ecliptical plane, from 0.3 to $1 \mathrm{AU}$ and for high-speed streams between 700 and $800 \mathrm{~km} \mathrm{~s}^{-1}$, Helios measured electron temperatures in the range $1.0-1.3 \times 10^{5} \mathrm{~K}$, with a power law $T_{e} \sim r^{-0.3}$, and proton temperatures in the range $2.3-2.8 \times 10^{5} \mathrm{~K}$, with a power law $T_{p} \sim r^{-0.8}$ [Marsch, 1991]. Data in the polar regions from Ulysses, from 1.5 to $3.2 \mathrm{AU}$, show slightly higher proton temperatures, in the range $2.6-3.2 \times 10^{5} \mathrm{~K}$, with $T_{p} \sim r^{-0.9}$ [Liu et al., 1995; Goldstein et al., 1996], and lower electron temperatures, from 7 to $7.5 \times 10^{4} \mathrm{~K}$, with $T_{e} \sim r^{-0.6}$ [Issautier et al., 1998].

Taking into consideration all these constraints, we have generated a set of functions which have a given starting value $T_{0}$ at $r=1 R_{\odot}$, then increase to a maximum value $T_{\max }$ at $r_{\max }$, and finally decrease monotonically to a given value $T_{E}$ at the Earth's orbit $r_{E} \simeq$ $215 R_{\odot}$, with a power law $T \sim r^{-n}$ as provided by the cited observations. The required functions are the product of an exponential, allowing the temperature to have a maximum, and of a power law, to give the observed behavior at large distances:

$$
T=T_{0}\left[\frac{e^{c(1-1 / x)}}{x}\right]^{\delta} .
$$

Here $x=x(r)$ must be chosen to be an increasing function of $r$ (normalized to unity at the coronal base), with a value $x=c$ at the location of the maximum (this results from imposing $T^{\prime}=0$ ) and with the expected power law, $T \sim r^{-n}$, at large distances:

$$
x(r)=1+(c-1) \frac{r^{n / \delta}-1}{r_{\max }^{n / \delta}-1} .
$$

Finally, the value of $c$ is to be determined by imposing $T=T_{\max }$ at $r=r_{\max } \Rightarrow x=c$, while the value of $\delta$, which controls the sharpness of the maximum, has to be adjusted to yield $T=T_{E}$ at $1 \mathrm{AU}$. A typical profile is shown in Figure 4.

In section 3.4 we will also show the effect of an extra peak in the temperature profile near the coronal base, in order to simulate the result of the heating, which is likely to be responsible for the enhanced density in plumes, as discussed in section 1.

\subsection{A Solution for the Fast Wind Model}

A two-stream fast solar wind model, which fits the observational constraints coming both from remote sensing measurements in the corona and data collected in situ in interplanetary space, is presented in this section. The comparison with data is mainly done for the interplume region, since it appears to be the dominant component (we will assume our usual value of $\alpha_{2}=5 \%$ for the filling factor of plumes in the coronal hole), and there is no clear identification yet of plumes in the solar wind.

To make our solution more realistic, we allow here for the presence of helium. If we indicate with $h$ the ratio of the abundance of helium (totally ionized) to that of hydrogen, it is easy to verify that the mean molecular weight may be written as $\mu=(1+4 h) /(2+3 h)$. Taking the value $h=5 \%$ measured in the fast solar wind, we get $\mu=0.56$, and the constants for normalization become $u_{\star}=122 \mathrm{~km} \mathrm{~s}^{-1}, \beta_{\star}=0.62$, and $g_{\star}=12.9$.

The temperature profile is chosen to be the same in the two regions, since the solution is very sensitive to differences in temperature and because we do not have clear proofs of such inhomogeneities (indirect measurements of electron and proton temperatures in the two regions are available only near the coronal base). In any case, the effects of possible differences in temperature will be shown in section 3.4. The function $T=T_{1}=T_{2}$ is chosen to have an initial value $T_{0}=0.5$ (in units of $10^{6} \mathrm{~K}$ ), a maximum $T_{\max }=3.8$ at $r_{\max }=3, \delta=0.35$, and $n=0.8$. If we assume an electron temperature around $10^{6} \mathrm{~K}$ in the inner corona, we have a peak of $6-7 \times 10^{6} \mathrm{~K}$ in the proton temperature, which is within the observational limit set by the new SOHO UV line-broadening data. The resulting temperature at the 

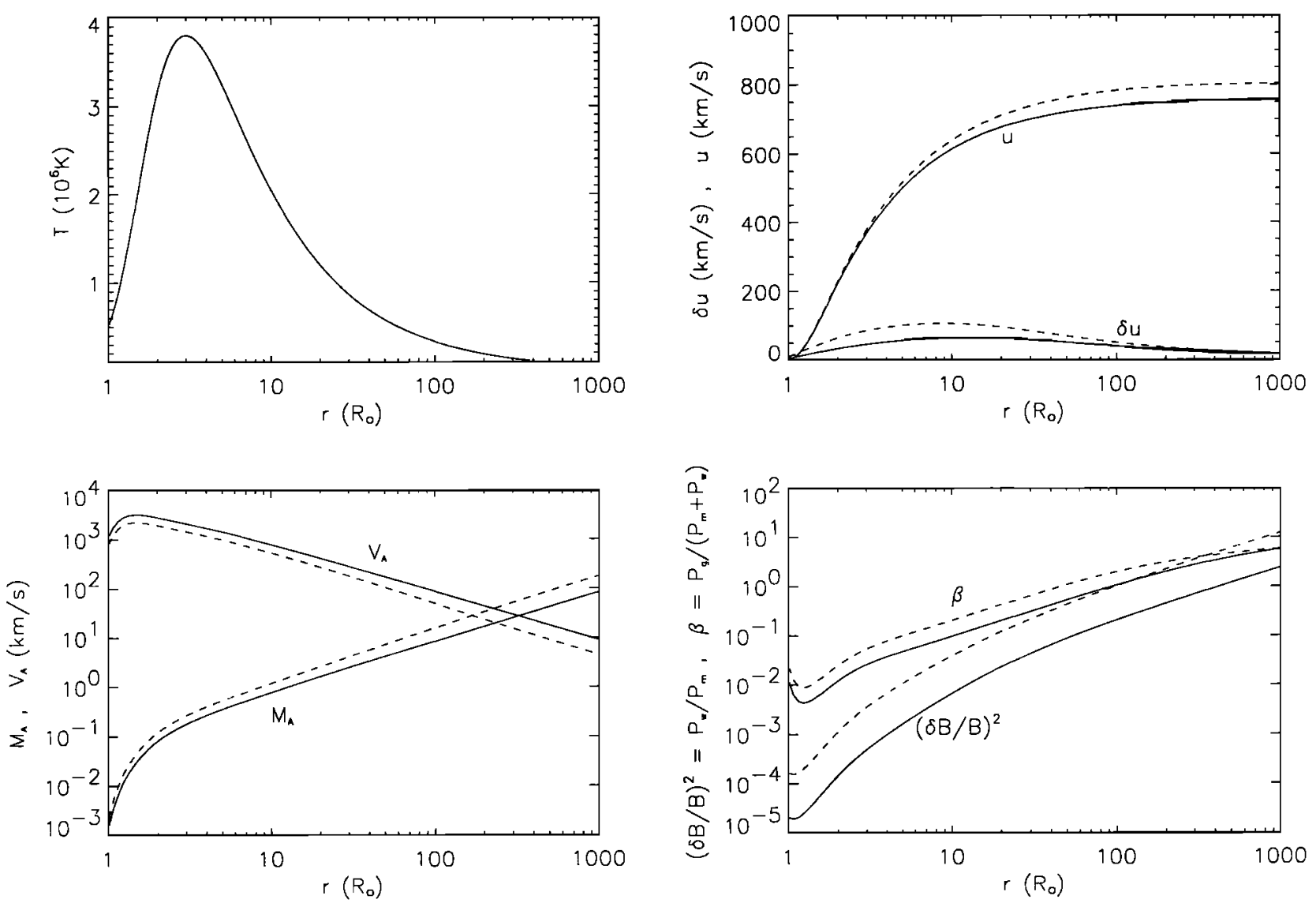

Figure 4. Various quantities resulting from the best fit fast wind model. The temperature profile is the same in both regions, and it is defined by equations (23) and (24), with the parameters given in the text. Nonspherical overall coronal hole expansion is assumed, with the parameterization given by Munro and Jackson [1977]. The plume is assumed to be 2 times denser than the interplume region and to have a higher flux of Alfvén waves. The precise parameters are given in the text. Following the same notation as that of section 3, solid lines refer to region 1 (interplume) and dashed lines refer to region 2 (plumes).

Earth is $1.8 \times 10^{5} \mathrm{~K}$, and it could correspond, for example, to an electron temperature $T_{e} \simeq 0.8 \times 10^{5} \mathrm{~K}$ and to a proton temperature $T_{p} \simeq 2.8 \times 10^{5} \mathrm{~K}$. By combining these values with the observed power laws $T_{e} \sim r^{-0.6}$ and $T_{p} \sim r^{-0.9}$, we get $T \sim r^{-08}$, which corresponds to the chosen value of $n$.

The effective temperature $T$ is plotted in Figure 4 from $r=1$ to $r=1000 R_{\odot}$, together with other quantities (both in the plume region and in the interplume region) resulting from the model. These are the bulk flow velocity $u$, the averaged Alfvén fluctuations in transverse velocity $\delta u$, the Alfvénic Mach number $M_{A}$ and speed $v_{A}$, the plasma $\beta$, and the ratio $(\delta B / B)^{2}$.

In this run a global superradial coronal hole expansion is considered, with the same analytical form and parameters as those discussed in the isothermal case. However, in order to minimize the effects of having $f_{\max }>1$ in the prediction of the values of the magnetic fields at the Earth, the base value in the interplume is extrapolated directly as

$$
B_{10}=B_{E} f_{\max } r_{E}^{2},
$$

with $B_{E}=3 \mathrm{nT}$ [Forsyth et al., 1996] and $B_{20}$ to be derived from pressure balance. Taking $n_{10}=4$ and $n_{20}=8$, with $T_{0}=0.5$, the fields at the base result both of the order of $\sim 10 \mathrm{G}$.

The velocity profile that results from the model has all the desired properties: low speeds near the base, strong acceleration within the first 10 solar radii (the critical points occur at $\simeq 2 R_{\odot}$ ), and final velocities in the observed range $750-800 \mathrm{~km} \mathrm{~s}^{-1}$. Since the velocity is almost constant after the acceleration phase and since the filling factors do not change largely $\left(a_{2}=1.7\right.$ at $1 \mathrm{AU})$, the rough average asymptotic behavior of the quantities can be predicted. For example, the number density goes as $n \sim r^{-2}$ (like the magnetic field), the Alfvénic Mach number goes as $M_{A} \sim r$, the Alfvén speed goes as $v_{A} \sim r^{-1}$, the plasma $\beta$ goes as $\beta \sim r^{2} T \sim$ $r^{2-n}$, and the wave pressure goes as $p_{w} \sim M_{A}^{-3} \sim r^{-3}$. These approximate asymptotic behaviors can be clearly seen in the plots of Figure 4. 


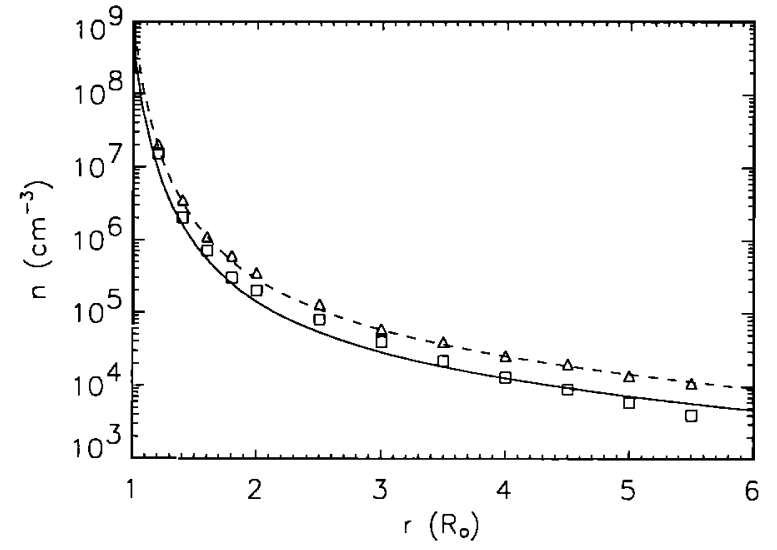

Figure 5. The resulting electron number densities in the inner corona compared with the white-light data by the Spartan spacecraft [Fisher and Guhathakurta, 1995]. The solid (dashed) line is the model prediction for the interplume (plume) region, whereas squares (triangles) are observational data for the corresponding region.

The average wave velocity fluctuation is taken to be larger in the plume by a factor 2,5 and $10 \mathrm{~km} \mathrm{~s}^{-1}$, respectively (yielding a factor 8 in the wave pressure, proportional to the energy per unit volume), in order to simulate the possible effect of reconnection dynamics at the base of the plume (provided waves are generated in the corona through this mechanism; if they were a direct consequence of random granular motion, then a uniform wave energy per unit volume would probably be more appropriate). This also enhances the velocity differences at $1 \mathrm{AU}$, yielding plumes that are faster than interplume regions. If, on the other hand, the same $\delta u$ is assumed and this is larger than a critical value, the interplume region turns out to be faster than the plume at large distances (provided that the temperature profiles are also the same). We will return to this point in section 3.4.

In Figure 5 the derived number densities, both for the plume plasma and for the interplume plasma, are compared with white-light data taken by the spaceborne Spartan 201-01 coronograph in the north polar hole in the range $1.16-5.5 R_{\odot}$ [Fisher and Guhathakurta, 1995; see also Habbal et al., 1995]. The good fit of our model with the data is obviously the result of a process of fine tuning of the various parameters, in particular, the temperature function and the base densities. The nonradial expansion of the coronal hole appears to play a minor role, provided the base magnetic fields are chosen by using (25).

The values of the most important physical quantities at the coronal base and at the Earth's orbit can be found in Table 1 for both regions. Note that pressure balance at large distances makes the density ratio, initially 2 , decrease to almost 1 with the consequent acceleration of the plasma inside plumes, as discussed for the isothermal case. Finally, note that the magnetic field ratio at
$1 \mathrm{AU}$ is basically given by $B_{1} / B_{2} \simeq a_{2}=1.7$, because this ratio is nearly 1 at the Sun, and the final plasma $\beta$ ratio is $\beta_{1} / \beta_{2} \simeq 0.65$, where the contribution of Alfvén waves is included in the total magnetic pressure.

\subsection{Parameter Study}

Because of uncertainties in the knowledge of some of the model parameters, especially those related to temperature profiles, it is necessary to study how the results of the model change when such values are modified. For example, we have so far used the same temperature profiles in the two regions, but it is important to have a basic understanding of how temperature differences influence plume-interplume dynamics. Let us neglect the effect of Alfvén waves, to simplify the problem, and study the case of plumes with slightly different temperatures.

All the parameters are the same as those in our best fit of section 3.3 , except the temperature maximum, which is increased to $4 \times 10^{6} \mathrm{~K}$ to compensate for the lack of wave acceleration $\left(\delta u_{1}=\delta u_{2}=0\right)$, and $\delta_{\mathbf{1}}=0.3$. Consider then two cases with different positions for the temperature maxima in the plumes, namely, $r_{2 \max }=1.5$ (case A) and $r_{2 \max }=5$ (case B). The power indexes are also changed to yield the same value of the temperature at the Earth, that is, $\delta_{2}=0.83$ and $n_{2}=0.6$ for $\mathrm{A}$ and $\delta_{2}=0.26$ and $n_{2}=0.9$ for $\mathrm{B}$, while the values of $T_{2}$ at $r=1$ and $r=r_{\max }$ are left unchanged. The results are shown in Figure 6 , where the temperature profiles, the normalized filling factors, the wind velocities, and the normalized number densities are plotted for the two cases.

The temperature profile has a large influence on the plasma parameters especially before the critical points.

Table 1. Values of the Various Quantities, Both in the Interplume and Plume Regions, at the Coronal Base and at $1 \mathrm{AU}$

\begin{tabular}{cccccc}
\hline & \multicolumn{2}{c}{ Interplume } & & \multicolumn{2}{c}{ Plume } \\
\cline { 2 - 3 } \cline { 5 - 6 } & $1 R_{\odot}$ & $1 \mathrm{AU}$ & & $1 R_{\odot}$ & $1 \mathrm{AU}$ \\
\hline \multirow{2}{*}{$a$} & 1.0 & 0.96 & & 1.0 & 1.70 \\
$\alpha a$ & 0.95 & 0.91 & & 0.05 & 0.09 \\
$B$ & $10.1 \times 10^{5}$ & 3.1 & & $10.0 \times 10^{5}$ & 1.8 \\
$n$ & $4.0 \times 10^{8}$ & 2.7 & & $8.0 \times 10^{8}$ & 3.1 \\
$u$ & 1.6 & 749 & & 1.7 & 795 \\
$n u$ & $6.6 \times 10^{5}$ & 2.0 & & $13.9 \times 10^{5}$ & 2.5 \\
$T$ & 5.0 & 1.8 & & 5.0 & 1.8 \\
$\delta u$ & 5 & 29 & & 10 & 34 \\
$(\delta B / B)^{2}$ & $2.1 \times 10^{-5}$ & 0.5 & & $16.7 \times 10^{-5}$ & 2.4 \\
$v_{A}$ & 1101 & 41 & & 774 & 22 \\
$M_{A}$ & $1.5 \times 10^{-3}$ & 18 & & $2.3 \times 10^{-3}$ & 36 \\
$\beta$ & $1.2 \times 10^{-2}$ & 2.1 & & $2.5 \times 10^{-2}$ & 3.2 \\
\hline
\end{tabular}

Here the magnetic field $B$ is expressed in $\mathrm{nT}$, the number density $n$ in $\mathrm{cm}^{-3}$, the velocities $u, \delta u$, and $v_{A}$ in $\mathrm{km} \mathrm{s}^{-1}$, the mass flux density $n u$ in units of $10^{8} \mathrm{~cm}^{-2} \mathrm{~s}^{-1}$, and the temperature in units of $10^{5} \mathrm{~K}$. 

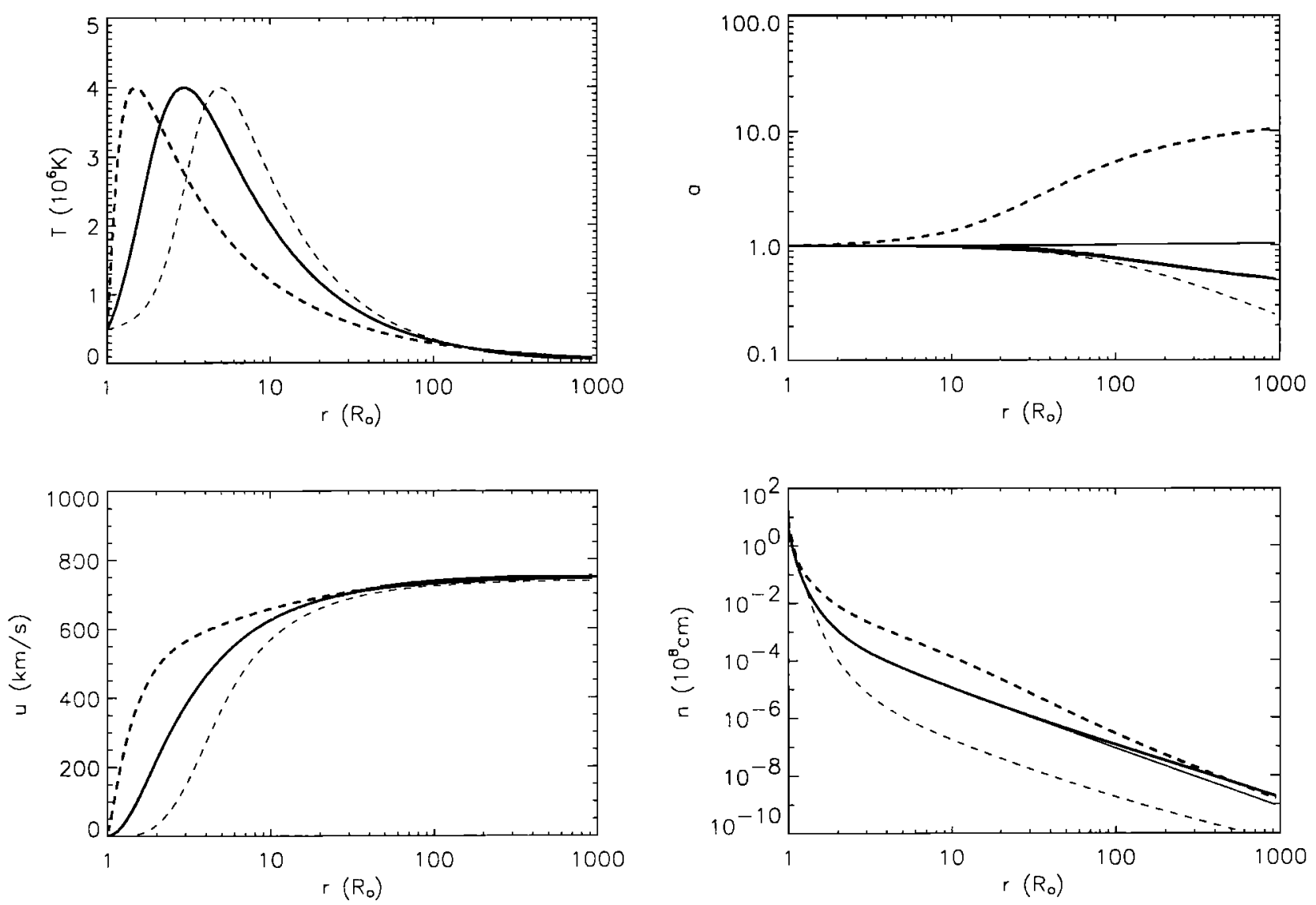

Figure 6. The effect of different plume temperature profiles. The parameters are the same as those in Figure 4, except some of those defining the temperature in the two regions: $T_{1 \max }=$ $T_{2 \max }=4 ; \delta_{1}=0.3 ; r_{2 \max }=1.5, \delta_{2}=0.83$, and $n_{2}=0.6$ for case $A$ (thick lines); and $r_{2 \max }=5.0$, $\delta_{2}=0.26$, and $n_{2}=0.9$ for case $\mathrm{B}$ (thin lines). Note that when the temperature maximum is closer to the Sun as compared with the interplume region, the plume expands superradially and is faster (case A), whereas the opposite holds for plumes with $r_{2 \max }>r_{1 \max }$ (case B).

As a matter of fact, the plume of case A contains a faster plasma in an expanding flux tube, whereas the opposite happens for the plume of case $\mathrm{B}$, and $T_{2}>T_{1}$ in the acceleration region for $\mathrm{A}$ while $T_{2}<T_{1}$ for $\mathrm{B}$. Thus a plume which is hotter than its surroundings contains a faster plasma only if this happens in the subsonic region. Another crucial result is that it is impossible to mantain a plume that is denser than the background at all heights if this is always cooler in the inner region: the reduced scale height makes the density drop very fast where the gravitation is stronger, no matter what the temperatures are at larger distances (case B; number density plot in Figure 6).

However, as it was pointed out in section 1, observations do seem to indicate that plumes should be cooler than the interplume. The question that first comes to mind is then how the dense material can be sustained against gravity, to mantain the observed density contrast with the interplume. The only answer, unless other unknown physical factors enter the momentum balance, hence appears to be the presence of a highertemperature region very near the base of plumes, as suggested by Wang [1994] as the result of a local enhanced heating. In Figure 7 we show how the presence of a secondary temperature peak near the base of the plume, superimposed to the same temperature profile, modifies the plasma parameters in the plume region: when the peak is present the plume is always denser than the background (Figure $7 \mathrm{~b}$ ), while without the peak the density contrast drops below unity (Figure 7a) as expected. Plumes are always slower because they are almost always cooler in the subsonic range showed here. Note, however, that the essential ingredient for the density behavior is not the secondary peak itself but rather the fact that plumes must be hotter at the coronal base, where most of the gravity stratification takes place. This is why all the peaks considered by Wang are above, if only slightly, the interplume temperature profile. A peak in $T_{2}$ with $T_{2}<T_{1}$ in the inner corona would never produce the desired result.

Another point of discussion is obviously the role of pressure balance, which is the main novelty as compared with previous wind/plume models. We have seen that under realistic conditions, the effects of pressure bal- 

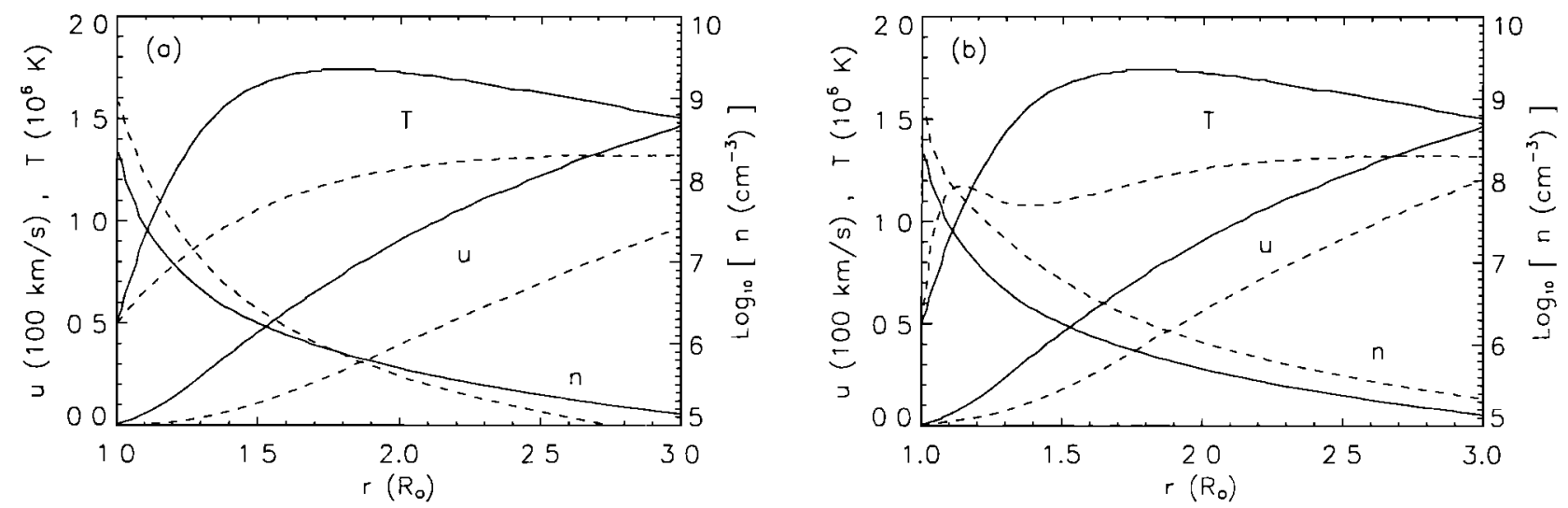

Figure 7. The modifications induced by a localized temperature enhancement near the base of plumes which are cooler farther out, including (a) the situation for a plume which is always cooler than the background plasma and (b) the effects of the temperature peak near the base (here the local maximum is taken at $1.1 R_{\odot}$ ), superimposed over the same temperature function. The base densities and some of the defining parameters of the temperature profiles $\left(T_{0}, T_{\max }\right.$, $r_{\max }$, and $T_{E}$ ) are taken by models CH1 and PL1 from Wang [1994], for the interplume (solid lines) and plume (dashed lines) regions, respectively. Note how the plasma inside plumes always remains denser than the background in Figure $7 \mathrm{~b}$, owing to the weaker gravity experienced where the plume is hotter.

ance are not very large when the two regions have the same temperature everywhere and that these are seen to appear only beyond $\simeq 10-20 R_{\odot}$. In any case, for a situation like case A of Figure 6, for example, where the temperatures have their maxima in different positions, pressure balance is fundamental in determining the relative velocities at the Earth: when rigid flux tubes are considered (no pressure balance is imposed), the plume is slower than the interplume; thus opposite results are found. This effect is clarified in Figure 8, where case A is plotted with and without the pressure balance condi-

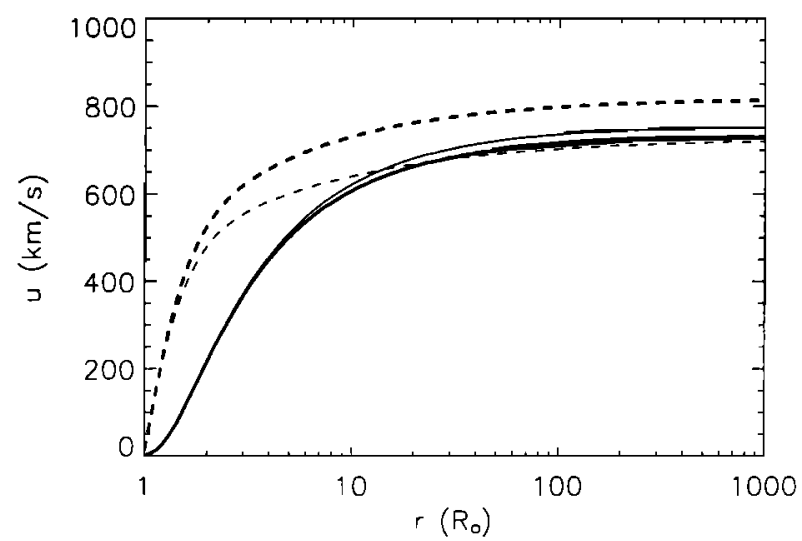

Figure 8. The effect of the pressure balance condition. The thick lines refer to the case A of Figure 6 with the pressure balance constraint, and the thin lines refer to the same model but ignoring the interaction of the flux tubes. Note the reversal of the asymptotic velocity ratios in the two cases. The effect has been enhanced here by choosing a higher initial density ratio $\left(n_{1_{0}}=3\right.$ and $\left.n_{20}=12\right)$ and lower magnetic fields $\left(B_{E}=1 \mathrm{nT}\right)$. tion (some of the values are changed in order to yield a higher $\beta$ in the plume to enhance the effect).

Finally, it is important to study the effect of having the same wave energy per unit volume (proportional to the wave pressure) both in plumes and in the interplume. To this purpose, let us use again our best fit parameters and take $\delta u_{20}=\delta u_{10} / \sqrt{n_{20} / n_{10}}$. As can be seen in Figure 9, which is to be compared with Figure 4 , the plume velocity is now smaller than the interplume velocity everywhere, if only slightly, and the same holds for the Alfvén average velocity fluctuation.

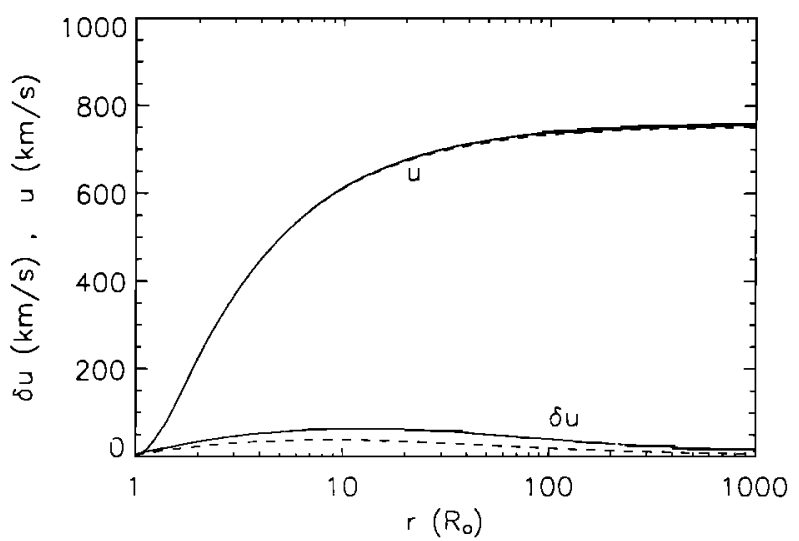

Figure 9. The bulk and average fluctuation velocities in the case of equal Alfvén wave energies (pressures) in the two regions. All the parameters are the same as those in Figure 4 , but now $\delta u_{20}=\delta u_{10} / \sqrt{2}$, that is, $\delta u_{10}=5$ and $\delta u_{20}=3.54$ (in $\mathrm{km} \mathrm{s}^{-1}$ ). Note that both $u_{2}$ and $\delta u_{2}$ are always smaller than the corresponding values in the interplume stream, opposite to what is shown in Figure 4, where the ratio in the initial wave energies was 8 . 
Note, however, that if the wave flux was taken smaller, then the plume would be faster, the limit being the case of no wave contribution discussed in section 2 .

\section{Conclusions}

We have described a 1-D solar wind model for the extension of a filamentary corona into space, in which the presence of dense ray-like structures (plumes) is considered self-consistently by taking into account lateral pressure balance (kinetic, magnetic, and wave pressures). This study is a first step to improve on earlier works [Wang, 1994; Habbal et al., 1995] where the plume and interplume were considered as two noninteracting regions. Since our main concern was to study in detail the interaction between the filaments and the background plasma, the energy balance (which requires in most of the cases the introduction of phenomenological heating functions in order to produce realistic results) has been neglected. Instead, a novel family of simple analytical functions has been introduced to describe the temperature profiles, which are to be given a priori in our treatment. The flexibility of such functions, for which the initial value at the coronal base, the position and value of the maximum, and the asymptotic value and gradient can be specified according to observational data, where available, makes it easy to study extensively the changes induced by different values of the single parameters.

The main result is that the temperature difference between the plume and its surroundings is the most important factor in the determination of the relative position of the critical points and of the expansion of the flux tubes, with resulting shear velocities and modifications of the other plasma parameters. We have seen that this is especially true when temperature differences occur before the critical points. Interesting effects, which are entirely due to the pressure balance constraint, are always present but emerge more clearly when we consider an isothermal plasma everywhere and in the absence of waves: plumes first undergo a slight contraction and then expand monotonically, with an increasing velocity shear (plumes are faster) as the kinetic pressure ratio drops toward unity.

In any case, mixing of the two streams owing to MHD Kelvin-Helmholtz instability appears to be possible only out to great distances from the Sun, say $\sim 100 R_{\odot}$, where the velocity shear becomes of the order of the local Alfvén speed ( $\mathrm{a}$ few tens of $\mathrm{km} \mathrm{s}^{-1}$ at $1 \mathrm{AU}$ ), in contrast to recent suggestions [Suess, 1998], according to which the mixing should already happen at $10-20 R_{\odot}$. This appears to be the case (see Figure 4) unless there are large temperature differences between the plume and interplume plasma, or if the temperature in the two regions peaks at very different radii (as shown by the example in Figure 6).

Another important result is that plumes cannot be cooler than the surrounding plasma at all $r$. In fact, in an almost hydrostatic situation such as that occurring in the lower corona, if plumes were cooler than the interplume region near the coronal base, their shorter local scale heights would induce a fast decay in the density contrast, with the result that plumes, defined as regions of greater density, would disappear near the coronal base, which is certainly not the case. This situation appears to be in contrast with recent measurements of both electron and (from line broadenings) ion temperatures [e.g., Wilhelm et al., 1998], according to which plumes actually seem to be always cooler. However, the SUMER data refer to heights above $1.03 R_{\odot}$, and more accurate measurements of the precise plasma parameters are required especially near the base of plumes, where a localized hotter region would explain naturally the enhanced densities observed.

The inhomogeneities predicted by our model in the interplanetary medium, remnants of coronal plumes signature, could have an observational counterpart in the so-called pressure balance structures and/or in microstreams, as mentioned in section 1 . The former are defined by an anticorrelation between the gas and magnetic pressures, while the other plasma properties have average fast streams' values; this is clearly in agreement with the defining characteristics of the present model, provided similar temperature profiles and wave energy fluxes are chosen in the two regions. On the other hand, microstreams, which can have both higher or lower velocity (approximately $\pm 40 \mathrm{~km} \mathrm{~s}^{-1}$ ), may be easily produced by changes in the flux of Alfvén waves at the coronal base, even when the temperature profiles are the same in the two regions: for realistic values of the wave energy flux, plumes result asymptotically slower if their flux at the base is the same as that in the interplume region or faster if this is larger by a certain critical amount. The observed absence of pressure balance in microstreams should then be explained, a possibility being the masking effects by the large variety of body and surface MHD waves that the dense filaments might carry.

When the temperature is nearly constant across the plume-interplume boundary, the filling factor of plumes typically increases only by $2-3$ times at $1 \mathrm{AU}$, as a result of their enhanced thermal pressure, but if plumes were indeed cooler even at large distances, the filling factor would be actually reduced, with plumes covering just a small fraction (a few percent) of the total coronal hole area. This could also provide an explanation to the first ionization potential (FIP) effect apparent paradox (see Del Zanna et al. [1998b] for a wider discussion). As a matter of fact, even if plumes were indeed characterized by a strong fractionation of heavy atoms with respect to photospheric values (with an enrichment of elements with a low first ionization potential as compared with those with a higher potential), only a small fractionation would be measurable in the polar solar wind anyway, as contributions from both regions are averaged together. This is an alternative view to that 
implying a disruption of the flux tube boundaries with consequent mixing of the plasma of plumes with the background. An attempt to measure the FIP effect in microstreams, as possible candidates for plumes in the fast wind, has been recently made by von Steiger $e t$ al. [1999], who analyzed data from the Ulysses SWICS experiment. The results are negative; that is no enhanced FIP effect is found in microstreams, suggesting that probably pressure-balanced structures are more likely to be the remnants of plumes in the heliosphere.

Concluding, we would like to point out that the validity of our thin flux tube model implies that the height of the coronal base must really be taken to be where the initial expansion of the quasi-potential flux concentrations has occurred, and the location of this height requires some discussion. If the flux is essentially unipolar, with small magnetic bipoles providing local neutral lines or points where plume activation occurs, this corresponds essentially to supergranule dimension, of order $30,000 \mathrm{~km}$. Above this height the expansion rate of plumes should be the same as the general coronal hole expansion rate, since the plasma $\beta$ is low in the inner corona. This is partially confirmed by the observations of DeForest et al. [1997], who measured the same linear expansion ratio of 3 , between 1 and $5 R_{\odot}$, both in a typical bright plume and in the coronal hole, but also measured an extra expansion of plumes beyond that height (where the general coronal hole expansion terminates), with a linear ratio of 6 in plumes at $15 R_{\odot}$. This strong superradial extra expansion of plumes, not observed by Fisher and Guhathakurta [1995], seems hard to model. The only way to obtain a normalized filling factor of $a_{2} \sim 4$ (corresponding to the cited linear ratio in plumes, which is twice that of the coronal hole at the same height) as close to the Sun as $15 R_{\odot}$ would be to have a hotter (and denser) plume expanding in a medium with $\beta \sim 1$, so that it is possible to overcome the collimating effects by the magnetic pressure, but this scenario contradicts the usual assumptions of cool plumes and a low- $\beta$ corona.

Acknowledgments. We would like to thank R. von Steiger, J. McKenzie, C. DeForest, S. Suess, P. C. Liewer, M. Neugebauer, and B. E. Goldstein for many stimulating discussions, and Y.-M. Wang and another referee for their useful comments. This work was completed while one author (M. V.) held a National Research Council Research Associateship at JPL.

Michel Blanc thanks Philippe Lemaire and another referee for their assistance in evaluating this paper.

\section{References}

Ahmad, I. A., and D. F. Webb, X-ray analysis of a polar plume, Sol. Phys., 58, 323, 1978.

Ahmad, I. A., and G. L. Withbroe, EUV analysis of polar plumes, Sol. Phys., 53, 397, 1977.

Alazraki, G., and P. Couturier, Solar wind acceleration caused by the gradient of Alfvén wave pressure, Astron. Astrophys., 13, 380, 1971.
Bohlin, J. D., N. R. Sheeley, and R. Tousey, Structure of the Sun's polar cap at wavelengths $240-600 \AA$, in Space Research $X V$, edited by $M$. J. Rycroft, pp. 651-656, Akademie, Berlin, 1975.

Corti, G., G. Poletto, J. L. Kohl, and G. Noci, Physical parameters in plume and interplume regions from UVCS observations, in Proceedings of the Fifth SoHO Workshop, Eur. Space Agency Spec. Publ., ESA SP-404, 289, 1997.

David, C., A. H. Gabriel, and F. Bely-Dubau, Temperature structure in coronal holes, in Proceedings of the Fifth SoHO Workshop, Eur. Space Agency Spec. Publ., ESA $S P$-404, 319, 1997.

DeForest, C. E., J. T. Hoeksema, J. B. Gurman, B. J. Thomson, S. P. Plunkett, R. Howard, R. C. Harrison, and D. M. Hassler, Polar plume anatomy: Results of a coordinated observation, Sol. Phys., 175, 393, 1997.

Del Zanna, L., A. W. Hood, and A. W. Longbottom, An MHD model for solar coronal plumes, Astron. Astrophys., 318, 963, 1997.

Del Zanna, L., A. W. Hood, M. Velli, and R. von Steiger, MHD models of coronal plumes, in Proceedings of Solar Jets and Coronal Plumes, Eur. Space Agency Spec. Publ., ESA SP-421, 359, 1998a.

Del Zanna, L., R. von Steiger, and M. Velli, The expansion of coronal plumes in the fast solar wind, Space Sci. Rev., $85,349,1998 b$.

Esser, R., S. R. Habbal, W. A. Coles, and J. V. Hollweg, Hot protons in the inner corona and their effect on the flow properties of the solar wind, J. Geophys. Res., 102, 7063, 1997.

Fisher, R., and M. Guhathakurta, Physical properties of polar coronal rays and holes as observed with the Spartan 201-01 coronograph, Astrophys. J., 447, 139, 1995.

Forsyth, R. J., A. Balogh, T. S. Horbury, G. Erdös, E. J. Smith, and M. E. Burton, The heliospheric magnetic field at solar minimum: Ulysses observations from pole to pole, Astron. Astrophys., 316, 287, 1996.

Geiss, J., et al., The southern high-speed stream: results from the SWICS instrument on Ulysses, Science, 268 , 1033, 1995.

Goldstein, B. E., M. Neugebauer, J. L. Phillips, S. Bame, J. T. Gosling, D. J. McComas, Y.-M. Wang, N. R. Sheeley, and S. T. Suess, Ulysses plasma parameters: Latitudinal, radial, and temporal variations, Astron. Astrophys., 316, $296,1996$.

Grall, R. R., W. A. Coles, M. T. Klinglesmith, A. R. Breen, P. J. S. Williams, J. Markkanen, and R. Esser, Rapid acceleration of the polar solar wind, Nature, 379, 429, 1996.

Habbal, S. R., R. Esser, and M. B. Arndt, How reliable are coronal hole temperatures deduced from observations?, Astrophys. J., 413, 435, 1993.

Habbal, S. R., R. Esser, M. Guhathakurta, and R. Fisher, Flow properties of the solar wind derived from a two-fluid model with constraints from white light and in situ interplanetary observations, Geophys. Res. Lett., 22, 1465, 1995.

Hassler, D. M., K. Wilhelm, P. Lemaire, and U. Schühle, Observations of polar plumes with the SUMER instrument on SOHO, Sol. Phys., 175, 375, 1997.

Hollweg, J. V., Alfvén waves in the solar wind: Wave pressure, Poynting flux, and angular momentum, J. Geophys. Res., 78, 3643, 1973.

Hu, Y. Q., R. Esser, and S. R. Habbal, A fast solar wind model with anisotropic proton temperature, J. Geophys. Res., 102, 14661, 1997.

Issautier, K., N. Meyer-Vernet, M. Moncuquet, and S. Hoang, Solar wind radial and latitudinal structure: Electron den- 
sity and core temperature from Ulysses thermal noise spectroscopy, J. Geophys. Res., 103, 1969, 1998.

Jacques, S. A., Momentum and energy transport by waves in the solar atmosphere and solar wind, Astrophys. J., 215 942, 1977.

Ko, Y.-K., L. A. Fisk, J. Geiss, G. Gloekler, and M. Guhathakurta, An empirical study of the electron temperature and heavy ion velocities in the south polar coronal hole, Sol. Phys., 171, 345, 1997.

Kohl, J. L., L. Strachan, and L. D. Gardner, Measurement of hydrogen velocity distributions in the extended solar corona, Astrophys. J., 465, 141, 1996.

Kohl, J. L., et al., First results from the SOHO ultraviolet coronagraph spectrometer, Sol. Phys., 175, 613, 1997.

Kopp, R. A., and T. E. Holzer, Dynamics of coronal hole regions, Sol. Phys., 49, 43, 1976.

Koutchmy, S., Study of the June 30, 1973 trans-polar coronal hole, Sol. Phys., 51, 399, 1977.

Liu, S., E. Marsch, S. Livi, J. Woch, B. Wilken, R. von Steiger, and G. Gloeckler, Radial gradients of ion densities and temperatures derived from SWICS/Ulysses observations, Geophys. Res. Lett., 22, 2445, 1995.

Marsch, E., Kinetic physics of the solar wind plasma, in Physics of the Inner Heliosphere 2: Particles, Waves and Turbulence, edited by R. Schwenn and E. Marsch, p. 45 , Springer-Verlag, New York, 1991.

McComas, D. J., B. L. Barraclough, J. T. Gosling, C. M. Hammond, and J. L. Phillips, Structures in the polar solar wind: Plasma and field observations from Ulysses, $J$. Geophys. Res., 100, 19893, 1995.

McComas, D. J., G. W. Hoogeveen, J. T. Gosling, J. L. Phillips, M. Neugebauer, A. Balogh, and R. Forsyth, Ulysses observations of pressure-balance structures in the polar solar wind, Astron. Astrophys., 316, 368, 1996.

McKenzie, J. F., M. Banaszkiewicz, and W. I. Axford, Acceleration of the high speed solar wind, Astron. Astrophys., $303,45,1995$.

McKenzie, J. F., W. I. Axford, and M. Banaszkiewicz, The fast solar wind, Geophys. Res. Lett., 24, 2877, 1997.

Munro, R. H., and B. V. Jackson, Physical properties of the polar coronal hole from 2 to $5 \mathrm{R}$, Astrophys. J., 213, 874, 1977.

Neugebauer, M., B. E. Goldstein, D. J. McComas, S. T. Suess, and A. Balogh, Ulysses observations of microstreams in the solar wind from coronal holes, J. Geophys. Res., 100, 23389, 1995

Parker, E., Dynamical properties of stellar coronas and stellar winds, III, The dynamics of coronal streamers, Astrophys. J., 139, 690, 1964.

Poletto, G., S. Parenti, G. Noci, S. Livi, S. T. Suess, A. Balogh, and D. J. McComas, Searching for coronal plumes in Ulysses observations of the far solar wind, Astron. Astrophys., 316, 374, 1996.

Saito, K., Polar rays of the solar corona II, Publ. Astron. Soc. Jpn., 17, 1, 1965.

Smith, E. J., A. Balogh, R. P. Lepping, M. Neugebauer, J. Phillips, and B. T. Tsurutani, Ulysses observations of lat- itude gradients in the heliospheric magnetic field: Radial component and variances, Space Sci. Rev., 72, 165, 1995.

Strachan, L., J. L. Kohl, H. Weiser, and G. L. Withbroe, A Doppler dimming determination of coronal outflow velocity, Astrophys. J., 412, 410, 1993.

Suess, S. T., Models of plumes: Their flow, their geometric spreading, and their mixing with interplume flow, in Proceedings of Solar Jets and Coronal Plumes, Eur. Space Agency Spec. Publ., ESA SP-421, 223, 1998.

Suess, S. T., G. Poletto, A.-H. Wang, S. T. Wu, and I. Cuseri, The geometric spreading of coronal plumes and coronal holes, Sol. Phys., 180, 231, 1998.

Thieme, K. M., E. Marsch, and R. Schwenn, Spatial structures in high-speed streams as signatures of fine structures in coronal holes, Ann. Geophys., 8, 713, 1990.

Velli, M., On the propagation of ideal, linear Alfvén waves in radially stratified stellar atmospheres and winds, Astron. Astrophys., 270, 304, 1993.

Velli, M., S. R. Habbal, and R. Esser, Coronal plumes and fine scale structure in high speed solar wind streams, Space Sci. Rev., 70, 391, 1994.

von Steiger, R., T. H. Zurbuchen, L. A. Fisk, and G. Gloeckler, Composition variations in fast solar wind streams, Proc. Sol. Wind 9 Conf., in press, 1999.

Walker, A. B. C., Jr., C. E. DeForest, R. B. Hoover, and T. W. Barbee Jr., Thermal and density structure of polar plumes, Sol. Phys., 148, 239, 1993.

Wang, Y.-M., Polar plumes and the solar wind, Astrophys. J., 435, 153, 1994.

Wang, Y.-M., and N. R. Sheeley, Coronal plumes and their relationship to network activity, Astrophys. J., 452, 457, 1995.

Wang, Y.-M., et al., Association of extreme-ultraviolet imaging telescope (EIT) polar plumes with mixed-polarity magnetic network, Astrophys. J., 484, 75, 1997.

Wilhelm, K., E. Marsch, B. N. Dwivedi, D. M. Hassler, P. Lemaire, A. Gabriel, and M. C. E. Huber, The solar corona above polar coronal holes as seen by SUMER on SOHO, Astrophys. J., 500, 1023, 1998.

Woo, R., and S. R. Habbal, Extension of coronal structure into interplanetary space, Geophys. Res. Lett., 24, 1159, 1997.

S. Casalbuoni, Physikalisches Institut, University of Bern, CH-3012 Bern, Switzerland.

(sara@lhc.lhep.unibe.ch)

L. Del Zanna and M. Velli, Dipartimento di Astronomia e Scienza dello Spazio, Università di Firenze, Largo E. Fermi 5, I-50125 Firenze, Italy.

(ldz@arcetri.astro.it; velli@arcetri.astro.it)

S.R. Habbal, Harvard-Smithsonian Center for Astrophysics, 60 Garden Street, Cambridge, MA 02138.

(shabbal@cfa.harvard.edu)

(Received June 22, 1998; revised January 7, 1999; accepted January 11, 1999.) 